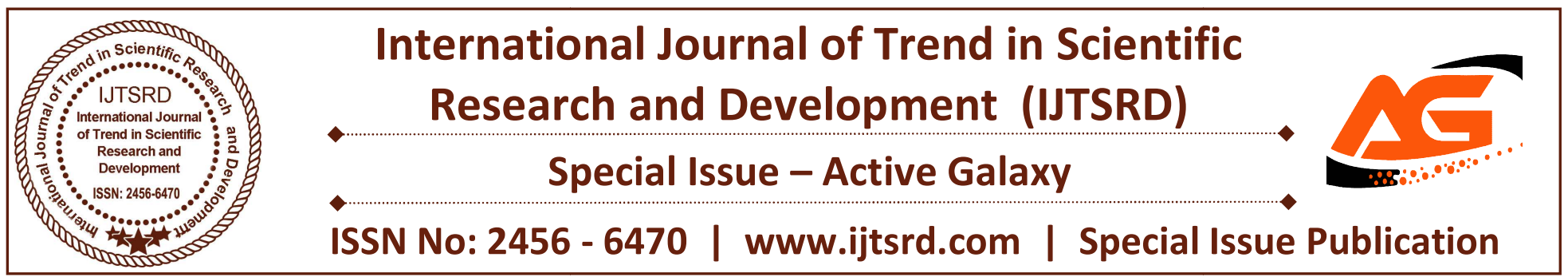

\title{
Real time Processor for the Authentication of Affected Herbal Medicinal Leaves Using Image Processing Techniques- Review
}

\author{
Debjyoti Guha $^{1}$, Hari Vishnu ${ }^{1}$, Mr. L. Jegan Antony Marcilin ${ }^{2}$, Mrs. T. Vijayashree ${ }^{3}$ \\ ${ }^{1}$ UG Student-ECE, ${ }^{2}$ Assistant Professor (ECE), ${ }^{3}$ Assistant Professor (E\&C) \\ Sathyabama Institute of Science and Technology, Chennai, Tamil Nadu, India
}

\section{ABSTRACT}

In biological sciences, herbal plants play a vital role in medicinal purpose. Identifying the leaf with unaffected leaves is more important nowadays. The leaves get affected due to many diseases like Phragmidium meuronatum, Cercospora jasmicola, Oidium tingitanium, Cephaleuros virescens. This paper focuses on the study and identification of unaffected leaves based on automated leaf recognition system. The proposed system uses image processing technique for image segmentation and feature extraction. The dataset includes 100 leaves of 10 different species. The leaves are compared with the database and the based on the threshold value the affected leaves are determined. The texture and color parameters are taken for disease authentication using image processing algorithm. Then it is fed into the real time processor Raspberry pi processor for which anywhere it can be used. The main use of Raspberry $\mathrm{Pi}$ is to make the system more user friendly. It uses four USB port and a real time camera which captures the image and feeds in the system. This work is more applicable in the field of agriculture and pharmaceutical industries.

\section{INTRODUCTION}

Agriculture is the backbone of our nation and Indian economy. The cultivation of crops for optimum yield and quality produce is highly essential. Plants become an important source of energy and only a primary source to the problem of global warming. A lot of research has been done on greenhouse agro systems and more generally on protected crops to control pests and diseases by biological means instead of pesticides. Disease and insect's pests are major problems in agriculture. In plants disease are found in various parts such as fruit, stem and leaves. The main disease of plants is viral, fungal and bacterial. If the farm is affected by a pest or a disease, it is to be rectified with immediate effect without any delay. But normally the farmers are not able to identify the actual disease deficiency. It can be done with the help of various Image processing techniques. India's diverse climate ensures availability of all varieties of vegetables. The diseases need to be controlled in the primary stage of the infection. The symptoms of diseases found on vegetable crops differ in color, shape, and size according to the cause. Plant disease is one of the crucial factor causes that reduces quantity and degrades quality of the agricultural products. The naked eye observation of experts is the main approach used in practice for detection and identification of plant diseases. But, this needs continuous monitoring of experts. Automatic detection of plant diseases is an important research topic as it may prove benefits in monitoring large field of crops, and thus automatically detect diseases from symptoms that appear on plant leaves. Comparatively, visual identification is less accurate and time consuming.

Section II deals with the literature survey which gives the prior and related work done earlier. Section III describes about the proposed Methodology which clearly explains the step by step procedure of the work to be carried out. Section IV narrates the conclusion of the review done with the leaf disease. 


\section{LITERATURE SURVEY}

Mrunalini et al. [1] presents the technique to classify and identify the different disease. The approach given in this for feature set extraction is the color cooccurrence method. For automatic detection of diseases in leaves, neural networks are used. The approach proposed can significantly support an accurate detection of leaf, and seems to be important approach, in case of steam, and root diseases, putting fewer efforts in computation. [2] This paper reports the results of experiments in improving performance of leaf identification system using Principal component Analysis (PCA). The system involved combination of features derived from shape, vein, color, and texture of leaf. PCA was incorporated to the identification system to convert the features into orthogonal features and then the results were inputted to the classifier that used Probabilistic Neural Network (PNN). This approach has been tested on two datasets, Foliage and Flavia, that contain various color leaves (foliage plants) and green leaves respectively. Samuel E. Buttrey et.al. [3] we construct a hybrid(composite) classifier by combining two classifiers in common use- classification trees and knearest-neighbor (k-NN). In our scheme we divide the feature space up by a classification tree, and then classify test set items using the k-NN rule just among those training items in the same leaf as the test item. This reduces somewhat the computational load associated with $\mathrm{k}-\mathrm{NN}$, and it produces a classification rule that performs better than either trees or the usual $\mathrm{k}-\mathrm{NN}$ in a number of well-known data sets. Rakesh Chaware et.al [4], present paper to detection of leaf diseases. In this used method is threefold: 1) identifying the infected object based upon k-means clustering; 2) extracting the features set of the infected objects using color co-occurrence methodology for texture analysis; 3 ) detecting and classifying the type of disease using NNs, moreover, the presented scheme classifies the plant leaves into infected and not-infected classes. Daniel Stanley Tan et.al. [5] Used k-means clustering algorithm to group the pixels into healthy and infected clusters. The clusters were then labeled and used to train an SVM classifier that would automatically determine which clusters contain infected pixels and which clusters contain healthy pixels. Advantage for existing system that it provides a feasible robust solution for detecting infected area segmentation of Cacao Pods. Harshal Waghmare et.al. [6], used Applications of Multiclass SVM are formulated for classification of disease identification which is observed in Grapes plants to make Decision Support Systems (DSS) automated and easily available for farmers. The system performs segmentation of a single leaf as input and analyses it through high pass filter to detect the diseased part of leaf. The advantage for existing system that it uses various feature extraction constraints to validate accuracy and efficiency. In work of G. Karafotias.et.al [7], researchers proposed Productivity of plant decreases due to infections caused by variety of diseases. The diseases not only restrict the growth of plant but also reduce quality and quantity of crop. Different techniques are adopted for detecting and diagnosis the diseases, but the better way is by using image processing. The author suggested a method in which initially the infected region is found then different features are extracted such as color, texture and shape. Finally, parameter classification technique is used for detecting the diseases. S. R. Dubey et.al. [8], presented the automatic detection of diseases present in the leaf image of plants. It is done with advancement of computer technology which helps in farming to increase the production. Mainly there is problem of detection accuracy and in neural network approach support vector machine (SVM) is already exist. The author discussed the various advantages and disadvantages of plant diseases prediction techniques and proposed a novel approach for detection algorithm. Kim et al. [9], have classified the grape fruit peel diseases using color texture features analysis. The texture features are calculated from the Spatial Gray-level Dependence Matrices (SGDM) and the classification is done using squared distance technique. Grape fruit peel might be infected by several diseases like cancer, copper burn, greasy spot, melanose and wind scar. Pydipati et al. [10], compared two different approaches to detect and classify three types of citrus diseases. The authors collected 39 texture features and created four different subsets of those features to be used in two different classification approaches. The first approach was based on a Mahalanobis minimum distance classifier, using the nearest neighbor principle. The second approach used radial basis functions (RBF) neural network classifiers trained with the back-propagation algorithm. According to the authors, both classification approaches performed equally well when using the best of the four subsets, which contained ten hue and saturation texture features. Jian et.al [11], proposed a method to recognize three kinds of cucumber leaf diseases. As in most approaches, the 
separation between healthy and diseased regions is made by a simple thresholding procedure. In the following, a variety of color, shape and texture features are extracted. Those features are submitted to an SVM with Radial Basis Function (RBF) as kernel, which performs the final classification. Abdullah et al. [12], tries to discriminate a given disease (corynespora) from other pathologies that affect rubber tree leaves. This algorithm does not employ any kind of segmentation. Instead, Principal Component Analysis is applied directly to the RGB values of the pixels of a low resolution $(15 \times 15$ pixels) image of the leaves. The first two principal components are then fed to a Multilayer Perceptron (MLP) Neural Network with one hidden layer, whose output reveals if the sample is infected by the disease of interest or not. H. Al-Hiary et al [13], have presented Otsu segmentation, K-means clustering and back propagation feed forward neural network techniques. Texture features could be extracted using Color Co-Occurrence methodology (CCM). Recognizing the disease NN (Neural Network) is used. They have tested on five diseases which are early scorch, Cottony mold, ashen mold, late scorch, tiny whiteness. Prakash M. Mainkar [14] Many techniques are used to determine the diseased leaves. Major techniques are K-means clustering, GLCM (grey level co-occurrence), $\mathrm{NN}$ classification and BPNN (free forward back propagation neural network). Sandesh Raut [15] Using step by step process like preprocessing, segmentation, K-means clustering, feature extraction using GLCM (grey level co-occurrence matrix) method and finally we use SVM (support vector machine) algorithm Sushil R. Kamalapurkar [16]. Thresholding and Back propagation network are used ANN(artificial neural network) are used for classification then PCA(principal component analysis) is used to reduce the number of neurons and dimension of data and increase speed pf $\mathrm{NN}$ (neural network).sometime threshold cannot be fixed and object in spot image cannot be located, Ranjeet Kaur and Miss Manjeet Kaur[17]. In this we use normally used techniques, but we use KNN (k-neural network) algorithm for comfortable cost and time consuming. S. Arivuazhagan [18] By using SGDMA (spatial grey level dependence matrix) and classified by square distance technique it is a statistical way to describe shape by sampling gray level occur in relation to other gray levels and co-occurrence features like contrast, energy, local homogeneity, shape are derived from co-occurrence matrix classification done in minimum distance criterion gives $86.77 \%$. the detection accuracy is improved to $94.74 \%$ by SVM classifier. Lakhir Kaur [19] there are many methods for the classification like KNN, PNN, genetic algorithm, SVM and PCA and modern technology called PB and they are classified based on morphological feature. Prajakta S. Garud [20] proposed a stand-alone system which will help to analyze plant disease very accurately within less time and fewer effort by raspberry pi steps involved feature extraction technique, $\mathrm{NN}$, diseases k-means algorithm. To detect plants using image processing technique. Shital Bankar [21] to identify diseased plants CBIR. Plant disease is detected by using histogram matching. The histogram matching is a color features and edge detection technique. The comparison technique between healthy sample of testing sample that is for the result for testing sample is diseased or not. Prof. Sanjay B. Dhaygude [22] the color transformation structure RGB is converted into HSV, Space between HSV is a good color descriptor. Then it undergoes the process then for texture analysis it goes under color co-occurrence matrix. Then the NN algorithm must improve the recognition and classification process. Sujeet Varshney et al. [23] segmentation is done, then RGB is changed HIS in-clustering process. Feature technology is used for more accuracy and method is used for finding the threshold value. SVM is used to increase the accuracy of detection. Lili N.A [25] propose an image noise detection and filtering algorithm-based Bayesian classification of combined with kernel regression framework that remove fixed value impulse noise of gaussian noise while preserving then recognition and classification algorithm modified hierarchical dynamic artificial neural network. Test image feature extraction and selection technique PCA, DA. Test image classification technique SVM and ANN technique. 


\section{PROPOSED METHODOLOGY}

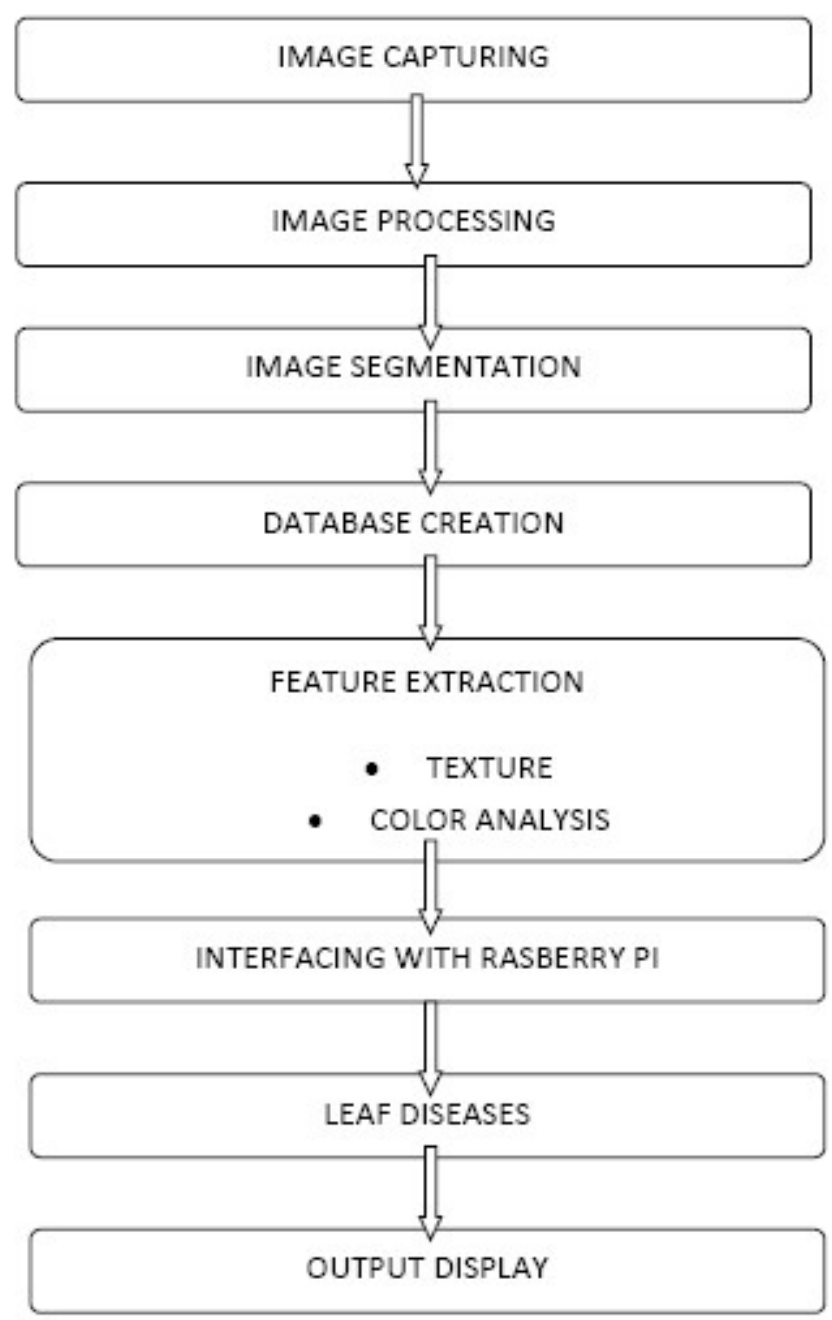

Fig 1: Proposed methodology

It is the first method of digital image processing. It captures the image through digital camera and stores it in digital media and it retrieves an image from hardware, so it can process further. Digital camera capture both affected and unaffected leaves. Then goes for image preprocessing it is to improve the image data unwanted distortion and enhance image features for further processing. Image segmentation is used to simplify the representation of an image into something meaningful and easier to analyze. K means clustering method a partition of image into clusters in which at least one part of cluster contain image with major area of diseased part. $\mathrm{K}$ means clustering algorithm is applied to classify the object into $\mathrm{k}$ number of classes according to set of features. Feature extraction is desire feature vector. It is the method for involving number of resources required to describe a large set of data accurately. Some texture analysis parameters are contrast, correlation, homogeneity, energy, Mean. Some color parameters are Kurtosis, skewness, standard deviation. The database contains the image of the several unaffected leaves of different species. The image is in JPEG format. An operating system is the set of basic programs and utilities that make your Raspberry Pi run. Software in Raspberry pi is Wolfram Mathematica. Mathematica is a computational programming tool. It is proprietary software that can be used on the Raspberry Pi and has been bundled with Raspbian and NOOBS. The main disease of plants is viral, fungal and bacterial. The viral disease is due to the viral changes in the environment conditions, the fungus is due to the presence of fungus in the leaves and the bacterial disease is due to the presence of germs in the leaf.

\section{CONCLUSION}

In the proposed project automatic identification of leaves gained more awareness in the current scenario and proves to be a very important in many areas including agriculture, forestry and pharmaceutical industries. This paper focused on the authentication of the unaffected leaves using image processing algorithm. Image processing techniques uses image preprocessing, feature extraction, color analysis and classification. In this paper image segmentation technique was used for identifying and also based on texture and color parameters the leaves were identified. A novel neural approach was trained for classifying the types of tulsi leaves using a modified Levenberg Marquadit algorithm and the efficiency was determined to be $68.8 \%$ which includes all the overall (texture, shape and color) parameters and individually produced the result of $92.2 \%$ efficiency. The experimental results provide a better accuracy compared to other existing system.

\section{ACKNOWLEDGMENTS}

We wish to express our sincere gratitude to our Pro Chancellor Madam Dr. Mariazeena Johnson and Vice President Dr.Marie Johnson for giving us the privilege to complete our work successfully. We would like to thank our School Dean and Head of the Department Dr.N.M.Nandhitha, for constantly supporting us on this project work. We wish to thank our project guide Mr.Jegan Antony Marcilin for encouraging us to do the work and giving us certain inputs when required. Finally we like to thank our parents and our friends. 


\section{REFERENCES}

1. Plant Disease Detection Techniques Using Canny Edge Detection \& Color Histogram in Image Processing by Shital Bankar, Ajita Dube, Pranali Kadam, Prof. Sunil Deokule (2014).

2. Plant Disease Detection in Image Processing using MATLAB by Sandesh Raut, Amit Fulsunge "International Journal of Innovative Research in Science, Engineering and Technology" in (2017).

3. A Review on Plant Leaf Classification and Segmentation "International Journal of Engineering and Computer Science" ISSN: 23197242, Volume 5 Issue 8 August 2016 by Lakhvir Kaur, Dr. Vijay Laxmi (2016).

4. Detection of plant leaf diseases using image segmentation and soft computing techniques in "INFORMATION PROCESSING IN AGRICULTURE" 41-49 journal homepage: www.elsevier.com/locate/inpa by Vijay Singh, A.K. Mishra (2017).

5. Detection of unhealthy region of plant leaves and classification of plant leaf diseases using texture features in "Agric Eng Int: CIGR Journal Open access" at http://www.cigrjournal.org by S. Arivazhagan, R. Newlin Shebiah, S. Ananthi, S. Vishnu Varthini (2013).

6. Digital image processing techniques for detecting, quantifying and classifying plant diseases in "Barbedo Springer Plus", HTTP 2/1/660 by Jayme Garcia, Arnal Barbedo (2013).

7. Detection of Diseases on Plant Leaf with the Help of Image Processing in "International Journal of Engineering Technology Science and Research" IJETSR, www.ijetsr.com,ISSN 2394 - 3386, Volume 4, Issue 8 by Prajakta S. Garud, Rajan Devi (August 2017).

8. Grading and Classification of Anthracnose Fungal Disease of Fruits based on Statistical Texture Features in "International Journal of Advanced Science and Technology" Vol. 52 by Jagadeesh Devdas Pujari, Rajesh Yakkundimath and Abdulmunaf Syedhusain Byadgi (March 2013).

9. Plant Leaf Disease Detection and Classification Using Image Processing Techniques in "International Journal of Innovative and Emerging Research in Engineering Volume 2, Issue 4" by Prakash M. Mainkar, Shreekant Ghorpade, Mayur Adawadkar (2015).
10. Advances In Image Processing For Detection Of Plant Diseases in "International Journal of Application or Innovation in Engineering \& Management (IJAIEM)" Web Site: www.ijaiem.org Email: editor@ijaiem.org, editorijaiem@gmail.com

Volume 2, Issue 11, by Smita Naikwadi, Niket Amoda (November 2013).

11. Plant Disease Prediction using Image Processing Techniques- A Review "International Journal of Computer Science and Mobile Computing" IJCSMC, Vol. 5, Issue. 5, May 2016, pg.394 398 by Sujeet Varshney Research Scholar CBS Group of Institution, Jhajjar, Haryana, Tarun Dalal assistant professor (CSE department) CBS Group of Institution, Jhajjar, Haryana (2016).

12. A Brief Review on Plant Disease Detection using in Image Processing "International Journal of Computer Science and Mobile Computing" IJCSMC, Vol. 6, Issue. 2, February 2017, pg.101 - 106 by Ranjeet Kaur Department of Electronics \& Communication Engineering, Rayat Bahra University, Mohali, India and Miss. Manjeet Kaur Assistant Professor, Department of Electronics \& Communication Engineering, Rayat Bahra University, Mohali, India (2017).

13. Review Paper on Identification of Plant Diseases Using Image Processing Technique "International Journal of Advanced Research in Computer Engineering \& Technology (IJARCET) Volume 3 Issue 11, by Rani Pagariya, Mahip Bartere (2014).

14. Detection of Plant Leaf Disease Using Image Processing Approach "International Journal of Scientific and Research Publications, Volume 6, Issue 2, February" by Sushil R. Kamlapurkar Department of Electronics \& Telecommunications, Karmaveer Kakasaheb Wagh Institute of Engineering Education \& Research, Nashik, India (2016).

15. Detection of Diseases on Plant Leaf with the Help of Image Processing "International Journal of Engineering Technology Science and Research IJETSR www.ijetsr.com ISSN 2394 - 3386 Volume 4, Issue 8" by Prajakta S. Garud Karmaveer Bhaurao Patil, Collage of Engineering, Satara. Shivaji University, Kolhapur Raja Devi. Karmaveer Bhaurao Patil, Collage of Engineering, Satara. Shivaji University, Kolhapur. 